This paper is published as part of Faraday Discussions volume 141:

Water - From Interfaces to the Bulk

Introductory Lecture

\section{Spiers Memorial Lecture \\ lons at aqueous interfaces \\ Pavel Jungwirth, Faraday Discuss., 2009 \\ DOI: $10.1039 / \mathrm{b} 816684 \mathrm{f}$}

Papers

The surface of neat water is basic

James K. Beattie, Alex M. Djerdjev and Gregory G. Warr, Faraday Discuss., 2009

DOI: $10.1039 / \mathrm{b} 805266 \mathrm{~b}$

Negative charges at the air/water interface and their consequences for aqueous wetting films containing surfactants

Katarzyna Hänni-Ciunel, Natascha Schelero and Regine von Klitzing, Faraday Discuss., 2009

DOI: $\underline{10.1039 / \mathrm{b} 809149 \mathrm{~h}}$

\section{Water-mediated ordering of nanoparticles in an electric field}

Dusan Bratko, Christopher D. Daub and Alenka Luzar, Faraday Discuss., 2009

DOI: $10.1039 / \mathrm{b} 809135 \mathrm{~h}$

Ultrafast phase transitions in metastable water near liquid interfaces

Oliver Link, Esteban Vöhringer-Martinez, Eugen Lugovoj, Yaxing Liu, Katrin Siefermann, Manfred Faubel, Helmut Grubmüller, R. Benny Gerber, Yifat Miller and Bernd Abel, Faraday Discuss., 2009

DOI: $\underline{10.1039 / \mathrm{b} 811659 \mathrm{~h}}$

Discussion

\section{General discussion}

Faraday Discuss., 2009

DOI: $10.1039 / \mathrm{b} 818382 \mathrm{c}$
Papers

Hydration dynamics of purple membranes

Douglas J. Tobias, Neelanjana Sengupta and Mounir

Tarek, Faraday Discuss., 2009

DOI: $10.1039 / \mathrm{b} 809371 \mathrm{~g}$

From shell to cell: neutron scattering studies of biological water dynamics and coupling to activity A. Frölich, F. Gabel, M. Jasnin, U. Lehnert, D. Oesterhelt, A. M. Stadler, M. Tehei, M. Weik, K. Wood and G. Zaccai, Faraday Discuss., 2009

DOI: $\underline{10.1039 / b 805506 h}$

Time scales of water dynamics at biological interfaces: peptides, proteins and cells

Johan Qvist, Erik Persson, Carlos Mattea and Bertil Halle, Faraday Discuss., 2009

DOI: $10.1039 / \mathrm{b} 806194 \mathrm{~g}$

Structure and dynamics of interfacial water in model lung surfactants

Avishek Ghosh, R. Kramer Campen, Maria Sovago and Mischa Bonn, Faraday Discuss., 2009

DOI: $10.1039 / \mathrm{b} 805858 \mathrm{j}$

The terahertz dance of water with the proteins: the effect of protein flexibility on the dynamical hydration shell of ubiquitin

Benjamin Born, Seung Joong Kim, Simon Ebbinghaus, Martin Gruebele and Martina Havenith, Faraday

Discuss. , 2009

DOI: $10.1039 / \mathrm{b} 804734 \mathrm{k}$

Discussion

General discussion

Faraday Discuss., 2009

DOI: $10.1039 / \mathrm{b} 818384 \mathrm{~h}$ 
Coarse-grained modeling of the interface between water and heterogeneous surfaces

Adam P. Willard and David Chandler, Faraday

Discuss., 2009

DOI: $\underline{10.1039 / \mathrm{b} 805786 \mathrm{a}}$

\section{Water growth on metals and oxides: binding,} dissociation and role of hydroxyl groups

M. Salmeron, H. Bluhm, M. Tatarkhanov, G. Ketteler, T. K. Shimizu, A. Mugarza, Xingyi Deng, T. Herranz, S. Yamamoto and A. Nilsson, Faraday Discuss., 2009

DOI: $\underline{10.1039 / b 806516 k}$

Order and disorder in the wetting layer on $\mathrm{Ru}(0001)$ Mark Gallagher, Ahmed Omer, George R. Darling and Andrew Hodgson, Faraday Discuss., 2009

DOI: $\underline{10.1039 / \mathrm{b} 807809 \mathrm{~b}}$

What ice can teach us about water interactions: a critical comparison of the performance of different water models

C. Vega, J. L. F. Abascal, M. M. Conde and J. L.

Aragones, Faraday Discuss., 2009

DOI: $\underline{10.1039 / \mathrm{b} 805531 \mathrm{a}}$

On thin ice: surface order and disorder during premelting

C. L. Bishop, D. Pan, L. M. Liu, G. A. Tribello, A. Michaelides, E. G. Wang and B. Slater, Faraday

Discuss., 2009

DOI: $\underline{10.1039 / b 807377 p}$

\section{Reactivity of water-electron complexes on} crystalline ice surfaces

Mathieu Bertin, Michael Meyer, Julia Stähler, Cornelius Gahl, Martin Wolf and Uwe Bovensiepen, Faraday

Discuss., 2009

DOI: $10.1039 / \mathrm{b} 805198 \mathrm{~d}$

Discussion

\section{General discussion}

Faraday Discuss., 2009

DOI: $\underline{10.1039 / \mathrm{b} 818385 f}$
Similarities" between confined and supercooled water

Maria Antonietta Ricci, Fabio Bruni and Alessia

Giuliani, Faraday Discuss., 2009

DOI: $\underline{10.1039 / \mathrm{b} 805706 \mathrm{k}}$

\section{Structural and mechanical properties of glassy} water in nanoscale confinement

Thomas G. Lombardo, Nicolás Giovambattista and Pablo G. Debenedetti, Faraday Discuss., 2009

DOI: $\underline{10.1039 / \mathrm{b} 805361 \mathrm{~h}}$

\section{Water nanodroplets confined in zeolite pores}

François-Xavier Coudert, Fabien Cailliez, Rodolphe Vuilleumier, Alain H. Fuchs and Anne Boutin, Faraday

Discuss., 2009

DOI: $10.1039 / \mathrm{b} 804992 \mathrm{k}$

Dynamic properties of confined hydration layers Susan Perkin, Ronit Goldberg, Liraz Chai, Nir Kampf and Jacob Klein, Faraday Discuss., 2009

DOI: $\underline{10.1039 / \mathrm{b} 805244 \mathrm{a}}$

Study of a nanoscale water cluster by atomic force microscopy

Manhee Lee, Baekman Sung, N. Hashemi and Wonho Jhe, Faraday Discuss., 2009

DOI: $\underline{10.1039 / \mathrm{b} 807740 \mathrm{c}}$

Water at an electrochemical interface-a simulation study

Adam P. Willard, Stewart K. Reed, Paul A. Madden and David Chandler, Faraday Discuss., 2009 DOI: $10.1039 / \mathrm{b} 805544 \mathrm{k}$

Discussion

General discussion

Faraday Discuss., 2009

DOI: $10.1039 / \mathrm{b} 818386 \mathrm{~b}$

Concluding remarks

Concluding remarks

Peter J. Feibelman, Faraday Discuss., 2009 DOI: $10.1039 / \mathrm{b} 817311 \mathrm{~g}$ 


\title{
Ultrafast phase transitions in metastable water near liquid interfaces
}

\author{
Oliver Link, ${ }^{a}$ Esteban Vöhringer-Martinez, ${ }^{a}$ Eugen Lugovoj, ${ }^{a}$ \\ Yaxing Liu, ${ }^{a}$ Katrin Siefermann, ${ }^{a}$ Manfred Faubel, ${ }^{b}$ \\ Helmut Grubmüller, ${ }^{c}$ R. Benny Gerber, ${ }^{d}$ Yifat Miller ${ }^{d}$ \\ and Bernd Abel ${ }^{* a}$
}

\author{
Received 8th July 2008, Accepted 9th July 2008 \\ First published as an Advance Article on the web 20th October 2008 \\ DOI: $10.1039 / \mathrm{b811659h}$
}

\begin{abstract}
Electron spectroscopy for chemical analysis (ESCA) is a powerful tool for the quantitative analysis of the composition and the chemical environment of molecular systems. Due to the lack of compatibility of liquids and vacuum, liquid-phase ESCA is much less well established. The chemical shift in the static ESCA approach is a particularly powerful observable quantity for probing electron orbital energies in molecules in different molecular environments. Employing high harmonics of $800 \mathrm{~nm}(40 \mathrm{eV})$, near-infrared femtosecond pulses, and liquid-water microbeams in vacuum we were able to add the dimension of time to the liquid interface ESCA technique. Tracing time-dependent chemical shifts and energies of valence electrons in liquid interfacial water in time, we have investigated the timescale and molecular signatures of laser-induced liquid-gas phase transitions on a picosecond timescale.
\end{abstract}

\section{Introduction}

Liquid-gas phase transitions in water are an everyday occurrence. Beyond equilibrium thermodynamics extreme states of matter, i.e. the dynamics of superheated metastable water are of fundamental as well of technical interest but the molecular picture in general is not well resolved., ${ }^{1,2}$

Liquid water has many unusual properties when compared with simple organic liquids and bulk water as well as single water molecules play a decisive role in many chemical ${ }^{3}$ and biological systems. ${ }^{4}$ The dynamical structure of water and the molecular picture of its important role in chemical and biological systems, often associated with a distinctive local structure of hydrogen bonds, ${ }^{5,6}$ has been studied with powerful spectroscopic techniques in the past ${ }^{7-10}$ however, this yielded a degree of understanding which is often insufficient. Water at extreme conditions (e.g. temperature and pressure) is also a field of continuous strong interest. ${ }^{11-13}$ In particular, water in systems at very high temperatures and pressures was investigated without providing general conclusive molecular pictures. Its hydrogen-bond structure has been investigated theoretically and experimentally. ${ }^{14}$ If water is heated

\footnotetext{
${ }^{a}$ Institut für Physikalische Chemie der Universität Göttingen, Tammannstrasse 6, 37077 Göttingen, Germany. E-mail: babel@gwdg.de; Fax: +49 (0)551 393150; Tel: +49 (0)551 393106

${ }^{b}$ MPI für Dynamik und Selbstorganisation Göttingen, Bunsenstrasse 10, 37073 Göttingen, Germany

${ }^{c}$ MPI für biophysikalische Chemie Göttingen, Am Fassberg 11, 37077 Göttingen, Germany

${ }^{d}$ Department of Chemistry, Hebrew University of Jerusalem, Israel
} 
above its critical point it forms a supercritical (sc) phase. Sc water plays an important role in many areas such as high-temperature chemistry, ${ }^{15}$ hazardous-waste treatment and possibly even the origin of life. ${ }^{16}$ The dynamics of water and solutes have been studied mostly in static cells with spectroscopic techniques in the time ${ }^{17}$ and frequency domains. ${ }^{15}$ Although the liquid-gas phase transition in water appears to be a trivial process in terms of thermodynamics, it is anything but trivial if one asks about the timescale of such a process, especially for superheated water, i.e. water heated significantly above its boiling point.

If short infrared pulses resonant on the water absorption of the $\mathrm{OH}$-stretch vibration at around $3 \mu \mathrm{m}$ are employed, it is possible to heat the water at a rate faster than the thermal expansion rate and to prepare extreme states of water, well above the boiling point and even significantly above the critical point. ${ }^{7}$ We will refer to these states as metastable, superheated and in the case of $T>T_{\text {critical }}=373.9{ }^{\circ} \mathrm{C}$ as supercritical. ${ }^{2}$ If water is prepared at these extreme states, the question is how the phase and the hydrogen-bonding network evolve in time. For water heated above its boiling point and below the critical point the system is expected to display a fast first-order phase transition (correlated with an enthalpy of transformation). ${ }^{2}$ The question to ask here is: how fast? Is it possible to observe a spectral signature in an ultrafast experiment that provides the timescale and the spectral signatures of such fast phase transitions, as well as information about the evolving hydrogen-bonding network? In addition, what is the dynamics of a system heated above the critical point? At the critical point the system should display a continuous phase transition, also called a second-order phase transition. Such a transition is not correlated with latent heat. ${ }^{1}$

In this contribution we report investigations of the molecular photoemission signature of the evolution of the phase and the hydrogen-bonding network of superheated water below and above the critical point, as well as its timescales. The

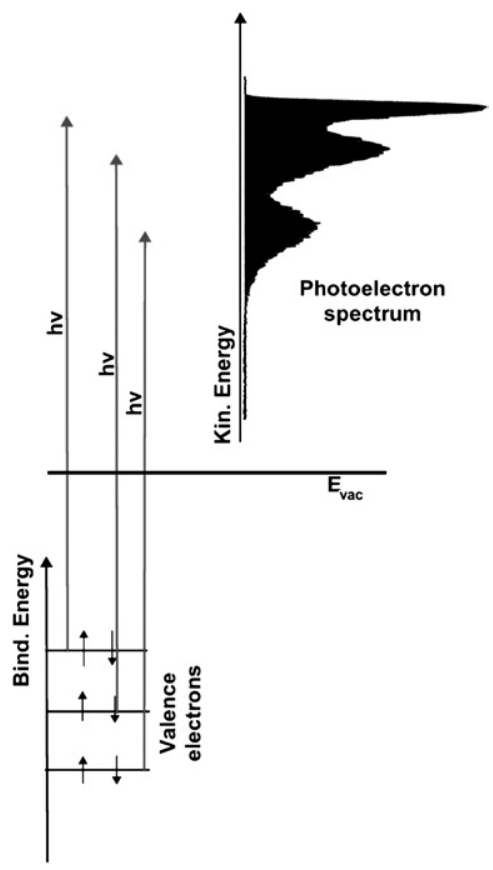

Fig. 1 Scheme illustrating the principle of photoelectron spectroscopy (high-energy photon produces an electron with a kinetic energy determined by the difference of the photon energy and the binding energy of the electron). 
combination of several powerful technologies, i.e. photoelectron spectroscopy near volatile liquid interfaces in vacuum, ${ }^{18}$ ultrafast pump-probe spectroscopy, and tabletop high-harmonics generation of soft X-ray radiation ${ }^{19}$ enabled us to add the dimension of time to the liquid interface ESCA (electronic spectroscopy for chemical analysis $^{\mathbf{2 0}}$ or liquid-phase photoelectron emission spectroscopy ( $c f$. Fig. 1).

\section{Experimental approach}

In the present experiments a commercial $\mathrm{Ti}$ : sapphire laser system (Hurricane, Spectra Physics) was employed, delivering short pulses with a pulse length of $100 \mathrm{fs}$ and a pulse energy of $1.3 \mathrm{~mJ}$ at a repetition rate of $1 \mathrm{kHz}$. The laser system employs the chirped pulse amplification (CPA) technique which incorporates typically four components, namely a $\mathrm{Ti}$ : sapphire oscillator, a grating stretcher, a regenerative amplifier and a grating compressor (Fig. 2). The internal laser setup was slightly modified by exchanging a mirror with a beamsplitter in front of the internal compressor. Three thirds of the internally amplified radiation were reflected by the beamsplitter and temporally compressed by the internal compressor to give $780 \mathrm{~mW}$
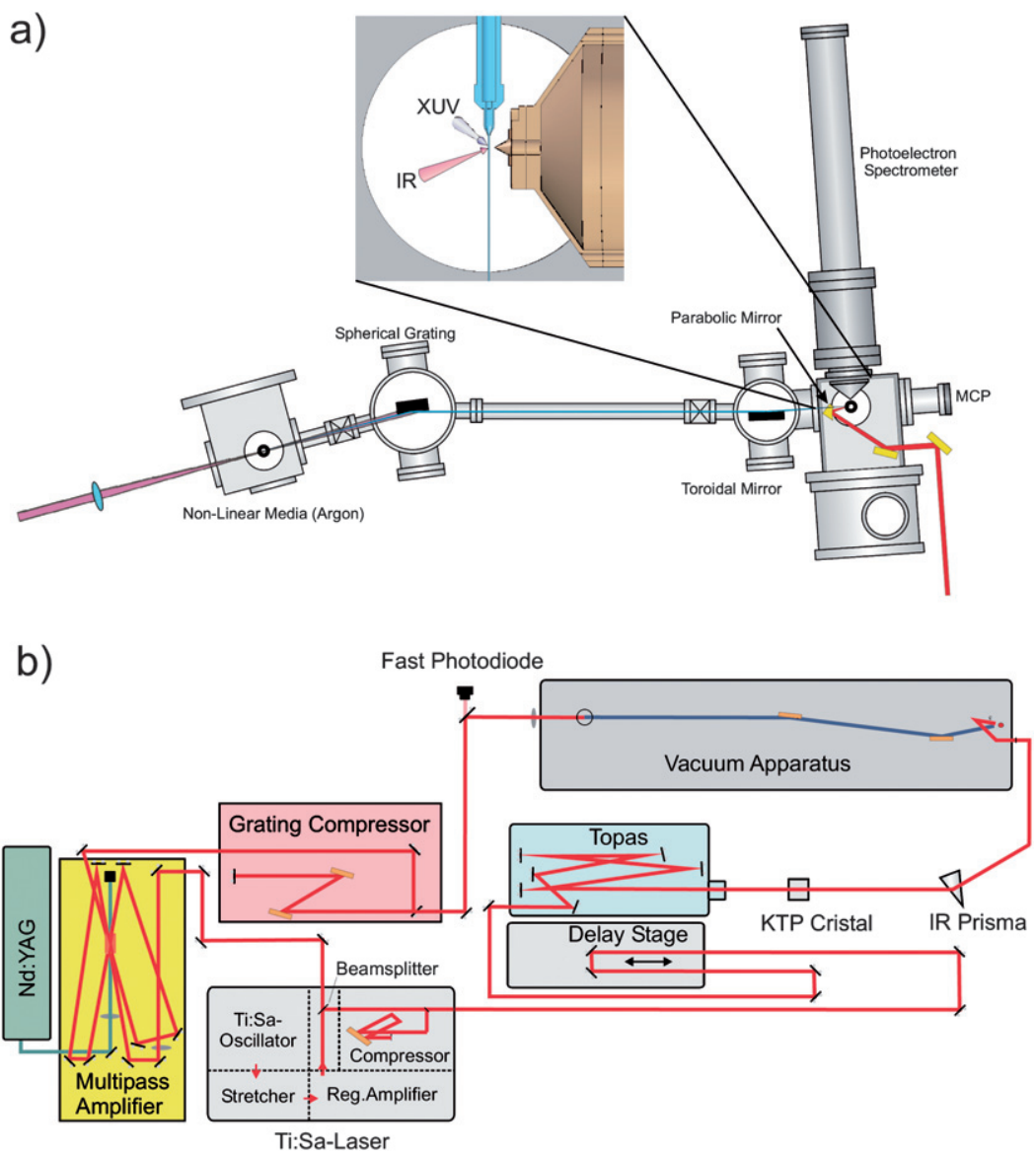

Fig. 2 (a) Schematic drawing of the vacuum apparatus consisting of four chambers and the TOF photoelectron spectrometer. In the inset the overlap of the IR-pump and the extreme ultraviolet or soft X-ray (EUV or XUV) probe pulse on the water filament is depicted. (b) Scheme of the optical setup, providing the IR-pump pulse $(2.6-3.0 \mu \mathrm{m})$ and the EUV-probe pulse $(39 \mathrm{eV})$. 
of the standard $100 \mathrm{fs}$ Hurricane output. The remaining $380 \mathrm{~mW}$ of the amplified but uncompressed pulses were transmitted and coupled out towards an external multipass amplifier. There an amplification up to $1.8 \mathrm{~W}$ takes place during three passes through an additional Ti : sapphire crystal (pumped by $10 \mathrm{~W}$ of an ORC 1000, Clark). Subsequent compression in an external grating compressor led to pulses of $1.2 \mathrm{~mJ}$ energy at $107 \mathrm{fs}$ pulse duration. The pulses are slightly longer than the standard Hurricane pulses due to gain-narrowing taking place in the multipass amplifier.

The $780 \mathrm{~mW}$ of the regular Hurricane output were directed to an optical parametric amplifier (TOPAS, Light Conversion) for photon conversion into the near infrared (Fig 2). As the standard model of the TOPAS only supports IR generation until $2.5 \mu \mathrm{m}$ due to the materials used, for extension of the wavelength region the TOPAS output was employed as the input for a second nonlinear process. In a subsequent KTP-crystal placed $15 \mathrm{~cm}$ behind the TOPAS exit an additional wave mixing process of the generated near IR around $1.1 \mu \mathrm{m}$ and the remaining fundamental radiation at $800 \mathrm{~nm}$ took place. ${ }^{21}$ Difference frequency generation and parametric amplification lead to radiation in the desired wavelength region at $2.6-3.0 \mu \mathrm{m}$. By optimizing the TOPAS settings pulse energies larger than $10 \mu \mathrm{J}$ could be obtained (up to $30 \mu \mathrm{J}$ at $2.7 \mu \mathrm{m}$ ). Finally a quartz prism (Infrasil) was used to separate the IR from the fundamental radiation and from other wavelengths resulting from the different wave-mixing processes.

The externally amplified $1.5 \mathrm{~mJ}$ pulses coming from the multipass amplifier are used for the generation of high harmonics. ${ }^{22,23}$ The essential peak power of $>10^{14}$ $\mathrm{W} \mathrm{cm}{ }^{-2}$ is obtained by focusing the short pulses with a $150 \mathrm{~mm}$ lens into an argon-filled capillary with a $2.3 \mathrm{~mm}$ inner diameter resulting in peak intensities of $8 \times 10^{14} \mathrm{~W} \mathrm{~cm}^{-2}$. The argon pressure inside the capillary is about 50-200 mbar. The radiation enters and leaves the capillary through laser-drilled holes which have a diameter of $100 \mu \mathrm{m}$. In order to optimize the high harmonic output the phase mismatch between the fundamental and the harmonic radiation in the interaction region must be minimized. ${ }^{24}$ This can be achieved by adapting the argon pressure and by tuning the parameters of the incident light in front of the focusing lens.

The vacuum chamber is pumped by a roots pump $\left(350 \mathrm{~m}^{3} \mathrm{~h}^{-1}\right)$ to remove the argon gas load from the chamber and keeping the background pressure at a level of $10^{-2}$ mbar in order to minimize the reabsorption of the XUV-radiation, which is less than $10 \%$ under the present conditions.

In the high-harmonics generation process several harmonics of the fundamental radiation are generated such that a dispersive element is necessary to separate a particular harmonic frequency. Therefore a spherical diffraction grating (700 lines per $\mathrm{mm}$ ) is illuminated at a deviation angle of $161.8^{\circ}$ exploiting the total external reflection. The grating has a radius of $17100 \mathrm{~mm}$ and is therefore not focusing but collimating the high-energy radiation. To protect the grating from thermal damage by the fundamental radiation a $150 \mathrm{~nm}$ aluminum filter can be introduced in the optical path in front of the grating. In this experiment the 25 th harmonic of the $800 \mathrm{~nm}$ radiation was used which corresponds to a wavelength of $32.1 \mathrm{~nm}$ and to a photon energy of $38.6 \mathrm{eV}$.

In the experimental chamber the IR-pump and the XUV-probe beam are overlapped in space and time on the liquid target (Fig. 2). The target is a liquid microjet which was introduced by Faubel and coworkers ${ }^{25-27}$ (Fig. 3). A flux of $0.3-0.4 \mathrm{ml}$ $\min ^{-1}$ of the solvent (i.e. water) is pressed through a micronozzle with a high-pressure liquid chromatography (HPLC)-pump (Economy 2/ED, Techlab Instruments). The nozzle diameter is in the range of $10 \mu \mathrm{m}$ giving a liquid filament in the experimental chamber of more or less the same diameter (Fig. 3). At velocities of 20$50 \mathrm{~m} \mathrm{~s}^{-1}$ the water filament remains continuous for about 2-3 mm until it breaks up into a stream of droplets (Fig. 3b) which is frozen out in a cooling trap with liquid nitrogen. A second cooling trap inside the chamber acts as a strong pump for gaseous water and allows in combination with a turbopump $\left(16001 \mathrm{~s}^{-1}\right)$ to keep the background pressure in the working apparatus at a level of $10^{-4} \mathrm{mbar}$. 

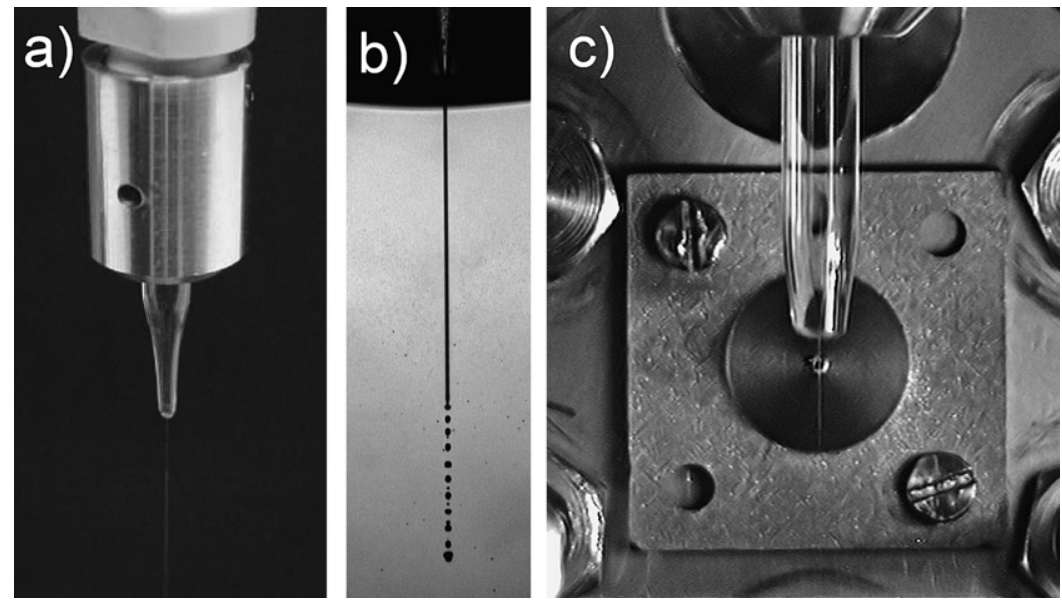

Fig. 3 (a) The quartz micronozzle producing a small water filament of $\approx 10 \mu \mathrm{m}$ diameter. (b) The stable filament and its decomposition into a stream of droplets after a certain length. (c) The micro water beam in front of the spectrometer entrance where a skimmer of $100 \mu \mathrm{m}$ diameter is mounted.

The IR-pump pulse is coupled into the experimental chamber via $\mathrm{a} \mathrm{CaF}_{2}$-window and focused onto the liquid filament with a $60^{\circ}$-off-axis parabolic mirror. The mirror has an aluminum surface and a focal length of $34 \mathrm{~mm}$ focusing the IR beam down to $70 \mu \mathrm{m}$ (somewhat larger than the filament's diameter). The nearly collimated XUVprobe beam is directed in vacuum, under grazing incidence, onto a toroidal mirror which has an imaging length of $2 f=360 \mathrm{~mm}$ ( $f$ being the focal length) ${ }^{28}$ The mirror is covered with gold, has a size of $20 \times 100 \mathrm{~mm}$, and radii of curvature of 24.1 and $5375.3 \mathrm{~mm}$ respectively. The focus dimensions and quality depend strongly on the incident angle and thus on precise pre-alignment. The focal point is slightly asymmetric with a focal diameter in the spectrometer plane of $100-120 \mu \mathrm{m}$. The XUV-radiation can be monitored with a XUV-sensitive CCD camera (OD2562Z, Proxitronic) which is installed on the optical axis on the backside of the liquid beam chamber (Fig. 2). Thus it is possible to see the filament's shadow in the XUV-image which is useful for alignment purposes.

For detection of the emitted photoelectrons a time-of-flight electron spectrometer (Kaesdorf) is mounted to the side (perpendicular to the beam and the optical axis) of the experimental chamber (Fig. 2). A skimmer of $100 \mu \mathrm{m}$ diameter separates the experimental chamber from the spectrometer and is part of a differential pumping stage towards a second inner aperture of $2 \mathrm{~mm}$ diameter. A vacuum better than $10^{-7}$ mbar can be maintained in the spectrometer under operation. The photoelectrons are ejected from the irradiated side of the liquid filament pointing towards the micro skimmer. Field-free conditions are maintained by a 3D Helmholtz coil construction to compensate for the earth magnetic field and other disturbing magnetic fields.

Under these conditions photoelectrons are sampled by the micro skimmer and propagate through the entrance of the spectrometer over a $60 \mathrm{~cm}$ long free-flight distance until they are finally accelerated $(6.9 \mathrm{~cm}, 280 \mathrm{~V})$ for higher detection efficiency towards the final MCP-detector. The $40 \mathrm{~mm}$ diameter MCP accepts a solid angle of $1.74 \mathrm{msr}$ of the initial electron emission. The resolution of the spectrometer in this liquid-beam photoemission configuration has been determined to be $0.2 \mathrm{eV}$.

If the pump and probe pulses are temporally overlapped, the spatial overlap on the beam can be detected (in the photoelectron spectrum) by an appearance of electrons with high kinetic energy generated and accelerated by a mixed 
IR-XUV-multiphoton absorption process. This effect (signal) can also be employed for cross-correlation measurements $(\approx 500 \mathrm{fs}$ for the present case) to estimate the pulse length of the XUV-probe pulse and the overall time resolution. ${ }^{29}$

\section{Computational methods}

\section{Molecular dynamics simulation}

All the simulations were carried out with the GROMACS software package. ${ }^{30,31}$ Thereby Newton's equations of motion were propagated with a time-step of $1 \mathrm{fs}$ using the SETTLE algorithm ${ }^{30}$ to constrain bond lengths and angles. The water model used in the present MD calculations was the SPC model. ${ }^{32}$ Test simulations employing other models (e.g. TIP4P) ${ }^{33}$ gave comparable results. The water box system with dimensions of $30 \times 4 \times 4 \mathrm{~nm}$ consisted of 16184 water molecules. It was equilibrated with a Berendsen thermostat and barostat at $300 \mathrm{~K}$ and 1 bar for $1 \mathrm{~ns}$. Afterwards the system was heated up to the required temperature and post-equilibrated for 200 ps at constant volume. To allow an expansion of the dense phase into vacuum, the water box was translated by $300 \mathrm{~nm}$ in the direction of the long edge and the total length of the box was elongated to $500 \mathrm{~nm}$. The phase transitions were simulated in a micro-canonical ensemble with the same time step for 100 ps. The short-range Coulomb interactions were calculated explicitly up to a distance of $1.2 \mathrm{~nm}$, and for the long-range interactions the particle mesh Ewald (PME) method was used. ${ }^{30}$

\section{Lifetimes of hot water aggregates}

The starting structure of the different water aggregates were taken from ab initio geometry optimization ${ }^{34}$ and minimized in the classical force field with the steepest descent algorithm to a convergence of $0.1 \mathrm{~kJ} \mathrm{~mol}^{-1}$. After minimization, each atom was assigned a random velocity from a Maxwell-Boltzmann distribution at 775K. Therefore, each atom was given a random velocity from a one-dimensional Maxwell distribution in each direction $(x, y, z)$ and the total kinetic energy of all atoms was calculated. This total kinetic energy with the number of degrees of freedom in the system was used to calculate the achieved temperature. The ratio of the achieved and the desired temperature $T$ served as scale factor for all velocities.

For the simulations a time step of $0.1 \mathrm{fs}$ for integration of Newton's equation was used and 101 trajectories with a total length of $100 \mathrm{ps}$ each were simulated. The position of the atoms were recorded every $2 \mathrm{fs}$. The lifetime of an aggregate was defined as the time after which one of the $\mathrm{O}-\mathrm{O}$ distances was longer than $4 \mathrm{~A}$ during at least $200 \mathrm{fs}$.

\section{Results \& discussion}

In the present experiments we used an ultrafast femtosecond near IR laser pulse $(2.5-3 \mu \mathrm{m})$ to excite water resonantly via its $\mathrm{OH}$-stretch vibration inducing subsequent ultrafast heating to internal energies between about 10 and $45 \mathrm{~kJ} \mathrm{~mol}^{-1}$ corresponding to hot transient phases between 450 and $1100 \mathrm{~K}$. The evolution of the superheated metastable water phase is probed with pulsed ultrafast photoelectron emission spectroscopy, employing EUV radiation produced via high-harmonics generation (HHG) and a liquid water jet in vacuum. ${ }^{25,35}$ The temperature as well as thermodynamic properties of the beam in vacuum can be characterized and controlled very well, as has been shown by us and others. ${ }^{25,27}$ In the present experiment only liquid water has been investigated. For the ultrafast liquid interface ESCA technique to work the infrared pump (near $3 \mu \mathrm{m}$ ) and the EUV (high harmonics) "laser-like" probe beam (near $40 \mathrm{eV}$ photon energy) have to be overlapped nearly collinearly in space and time on the microjet. The EUV probe beam alone in the present case ionizes water and produces photoelectrons from the ${ }^{1} b_{1},{ }^{3} a_{1},{ }^{1} b_{2}$, 
and ${ }^{2} a_{1}$ ( $c f$. Fig. 4, at negative delays) valence electrons of liquid water and some gas-phase water around the liquid microbeam. ${ }^{25,36}$ Photoelectrons near the liquid interface (about 3 monolayers of water) can escape into vacuum nearly unperturbed $^{37}$ and can be sampled with a $100 \mu \mathrm{m}$ (micro) skimmer at a distance close to the microbeam $(100-500 \mu \mathrm{m})$ and their kinetic energy can be measured in a time-of-flight electron spectrometer. The electron spectrometer, differentially pumped between skimmer and spectrometer, enables us to record the entire photoelectron spectra (multiplex advantage) and a reference spectrum at negative delay times in single shots at $1 \mathrm{kHz}$ repetition rate that are averaged for predefined time-steps of the delay of the two femtosecond (fs) pulses. The time-of-flight data are converted into kinetic energies of the photoelectrons and in turn into binding energies of the valence electrons depending upon local intramolecular electron densities and the molecular environment (e.g. hydrogen bonding). With this technology, time-dependent photoelectron spectra as a function of delay time between the IR-pump and the EUV probe pulses have been recorded. A time-series of spectra (with perpendicular polarization of pump and probe beams) is displayed in Fig. 4.
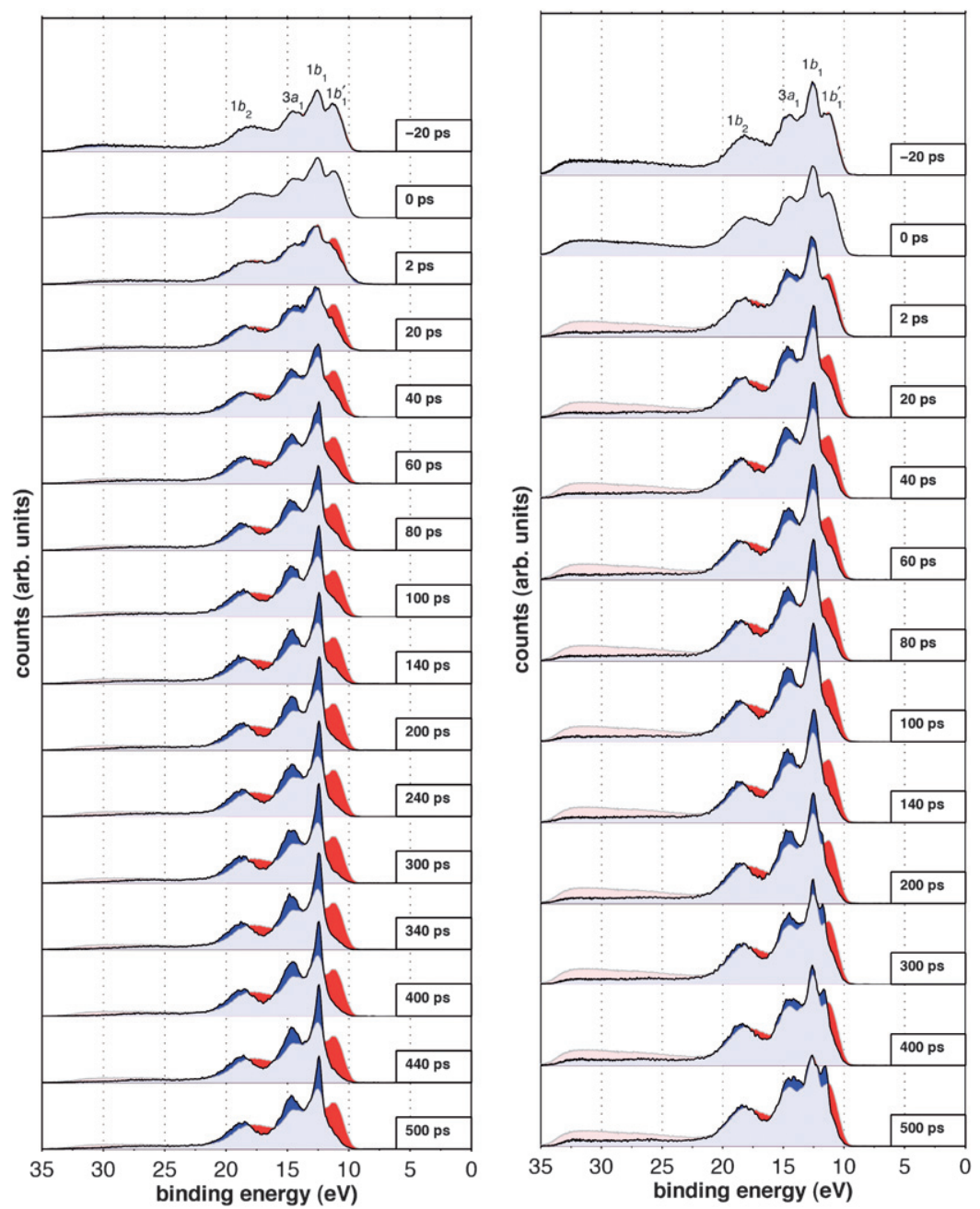

Fig. 4 Time-resolved photoelectron spectra of liquid and metastable water (2700 nm: left and $2900 \mathrm{~nm}$ : right). 
The broad features of the ${ }^{1} b_{1},{ }^{3} a_{1},{ }^{1} b_{2}$ and ${ }^{1} b_{1, \text { liq. }}$ photoelectrons can be resolved. Prominent features of a photoelectron spectrum of water for the valence electrons are known and easily assignable to the ${ }^{1} b_{1},{ }^{3} a_{1}$ and ${ }^{1} b_{2}$ orbitals of isolated water in the gas phase. ${ }^{25,36}$ In the present case a superposition of spectra from the liquid and the gas phase of water is observed. While somewhat narrower photoemission lines of the ${ }^{1} b_{1},{ }^{3} a_{1}$ and ${ }^{1} b_{2}$ electrons in the gas phase are overlapped with shifted (towards lower binding energies) and broader ${ }^{1} \mathrm{~b}_{1, \text { liq. }},{ }^{3} \mathrm{a}_{1 \text {,liq. }}$ and ${ }^{1} \mathrm{~b}_{2 \text {,liq }}$ lines of liquid water, the ${ }^{1} b_{1, \text { liq. }}$ photoemission line is resolved best because it is the line with the largest liquid-gas shift of all valence orbitals and observable in a region of least overlap. ${ }^{25,38}$ The upper limit of the energy resolution in the photoelectron spectra has been measured for the narrowest lines to be $0.2 \mathrm{eV}$ at present.

For clarity, the time-resolved spectra in Fig. 4 (solid lines, colored in blue) have been plotted with a color code displaying the differences to the reference spectrum at negative delays. Red displays a decrease and blue an increase in intensity relative to the reference spectrum. All spectra are normalized on a shot-to-shot basis to the corresponding reference spectra at negative delays and averaged until a satisfactorily signal-to-noise ratio is obtained. Usually, 100000 shots are averaged for one time point. This procedure enabled us to eliminate shot-to-shot variations and energy drifts of the high harmonic radiation and the pump pulses. Tracing time-dependent chemical shifts and binding energies of valence electrons in superheated water in time, we have investigated the timescale and molecular signatures of the evolution of superheated water (with energy contents varied via the known absorption coefficient of water and the wavelength of the tunable IR-laser, see ref. 13 and references cited there) on a picosecond timescale with a time-resolution of about $500 \mathrm{fs}$ (to be improved soon via multilayer mirrors ${ }^{39}$ or a second grating ${ }^{40}$ ). If intensities at a particular chemical shift (i.e. binding energy) are monitored, time-profiles can also obtained instead of time-dependent spectra.

The positions (binding energies) of particular interest in the spectrum belong to: the low-energy wing of the (static) liquid ${ }^{1} b_{1 \text {,liq. }}$ photoemission line $(10.5 \mathrm{eV})$, its maximum $(11.16 \mathrm{eV}),{ }^{38}$ a position between the ${ }^{1} b_{1}$ and the ${ }^{1} b_{1, \text { liq. }}$ emission maximum $(11.6 \mathrm{eV})$ and the ${ }^{1} \mathrm{~b}_{1}$ gas-phase emission maximum $(12.6 \mathrm{eV})$. While the energy windows at $10.5 \mathrm{eV}$ and at $11.16 \mathrm{eV}$ both monitor the decay of the liquid phase, the $11.6 \mathrm{eV}$ energy monitors the appearance of the supercritical phase and the presence of clusters and water aggregates at later times. The chemical shift at $12.6 \mathrm{eV}$ monitors the increase of gas-phase water. As we watch the spectra as a function of time (e.g. in Fig. 4, left panel), the initial shift towards higher binding energy and a decay of the liquid ${ }^{1} b_{1, \text { liq. }}$ peak at $11.16 \mathrm{eV}$ is obvious (red). At the same time an increase of the ${ }^{1} b_{1}$ gas-phase peak at $12.6 \mathrm{eV}$ occurs (blue). Beyond these changes also intensity at 14.5 and $18 \mathrm{eV}$ builds up which belongs to the gas-phase photoemission peak of the ${ }^{3} a_{1}$ and ${ }^{1} b_{2}$ electrons of water. At $17 \mathrm{eV}$ intensity of the ${ }^{1} b_{2}$,liq. orbital vanishes as a function of time. These overall features (initial shift and decay of the liquid-phase photoemission) is a clear signature for a fast evaporation of the liquid after excitation with an IR-femtosecond pulse resonant with the $\mathrm{OH}$-stretch of water in the liquid phase. The internal energies of the excited water phase have been estimated from the known absorption coefficients of water ${ }^{13}$ and the penetration depth and have been determined to be $\approx 10$ and $\approx 45 \mathrm{~kJ} \mathrm{~mol}^{-1}$ corresponding to $\approx 450$ and $\approx 750 \mathrm{~K}$ respectively. While the first traces of Fig. 4 (left panel) correspond to temperatures of $450 \mathrm{~K}$ (superheated but below the critical temperature $T_{\text {crit. }}$ ) the right panel displays experiments for $750 \mathrm{~K}$ corresponding to a supercritical fluid. Beyond the overall features discussed above a new feature is transient intensity in a range of the chemical shift (i.e. at $11.6 \mathrm{eV}$ ) between the liquid-phase ${ }^{1} b_{1, \text { liq. }}$ peak at $11.16 \mathrm{eV}$ and the gas-phase peak at $12.6 \mathrm{eV}$. It is known that in this energy range photoemission of clusters occurs $\left({ }^{1} b_{1}\right) \cdot{ }^{41,42}$ These features and trends are easily observed in the time-resolved traces of the chemical shift as a function of time (Fig. 4). For excitation at $2700 \mathrm{~nm}$ the intensity at $10.5,11.16$ and $11.6 \mathrm{eV}$ is decreasing with a fast component within 5-10 ps and a slower component on a $500 \mathrm{ps}$ timescale. At the same time 
the gas-phase photoemission of the ${ }^{1} b_{1}$ at $12.6 \mathrm{eV}$ corresponding to gas-phase water increases. For excitation at $2900 \mathrm{~nm}$ near the maximum of the liquid-water absorption the intensity at 10.5 and $11.16 \mathrm{eV}$ decreases on a faster (5-10 ps) and then apparently slower (but overall still fast) timescale of 100-150 ps. Contrary to the previous case, intensity increases again in the intermediate range of $11.6 \mathrm{eV}$ for longer times while the gas-phase intensity at $12.6 \mathrm{eV}\left({ }^{1} \mathrm{~b}_{1}\right)$ first increases and then decreases again (cf. Fig. 4, right panel). We attribute this feature tentatively to the appearance of new species, namely hot water aggregates or hot clusters, between the liquid and gas phases from a dispersion of the liquid and/or an ultrafast re-condensation of hot gas-phase molecules.

The preliminary conclusion from the experiment is that a superhot sub-critical or supercritical phase is formed with a characteristic spectral signature, i.e. an early shift towards higher binding energies. The origin of this chemical shift may be attributed to a weakening of the hydrogen-bonding network and the interaction being correlated with fewer hydrogen bonds per water molecule. Depending upon the energy content of the hot water phase, (i) the liquid disappears in a process that may be called fast evaporation or (ii) the liquid is dispersed. In the former case the loss of the liquid phase and the gain of the gas phase is irreversible. In the supercritical case the liquid-phase photoemission vanishes and the gas phase builds up temporarily. On a timescale of a few hundred picoseconds the gas phase vanishes again and photoemission in the $11.6 \mathrm{eV}$ energy range monitors more or less large clusters $^{41}$ formed either from the disintegrating liquid or through re-condensation of the gas phase or both, when aggregates from the disintegrating liquid serve as nucleation seeds. The disappearance of the gas phase may suggest the latter scenario at later times. The experiment shows unambiguously that a superheated sub-critical and supercritical water phase have a finite lifetime and that the spectral features and its timescales can be observed with time-resolved ESCA. While the evolution of the sub-critical phase reresembles fast (normal) evaporation, the evolution of the supercritical phase results in a very fast dispersion of the fluid into a distribution of monomers and hot aggregates. This effect is gradually observed when changing the energy content of the water through changing the excitation wavelength of the IR laser from 2650 to $2900 \mathrm{~nm}$ (maximum of absorption). It must be kept in mind that in the present experiment mixed spectra of gas phase and liquid phase are always observed. It is also important to note that we observe approximately the same near-interface molecules (initially in the first three monolayers ${ }^{37}$ of the liquid and then in the dense, evolving gas phase) throughout the evaporation process, i.e. the escape depth of electrons observed in our experiment depends upon the number density perpendicular to the water surface.

In order to obtain more insight into the processes the time-resolved data have been compared with molecular dynamics simulations of the process (evolution of a hot near-interface water phase for different internal energies).

The simulations are carried out with the GROMACS software package and the details are given in the computational methods section. For different times the evolution of the (extremely) heated water phase is displayed in Fig. 5. The water molecules are color-coded in such a way that the color corresponds to the thermodynamic state in the schematic phase diagram of water (shown in the inset) if thermodynamic equilibrium is assumed. Under this assumption the local density and the kinetic energy of each molecule was calculated and averaged over 2 ps. With the temperature calculated from the kinetic energy of the molecules (rigid SCP model for water), the pressure was calculated from the local density employing an extended equation of state for liquid water. ${ }^{43}$ According to the pressure and temperature the assignment according to the phase diagram was made. From the simulation we see that the evolution of the superheated phase is mainly an expansion of a supercritical phase for quite some time. However, the density is quickly changing and the phase is obviously heterogeneous, which manifests itself in the presence of various transient aggregates, which form and partially dissociate again on a picosecond timescale. 


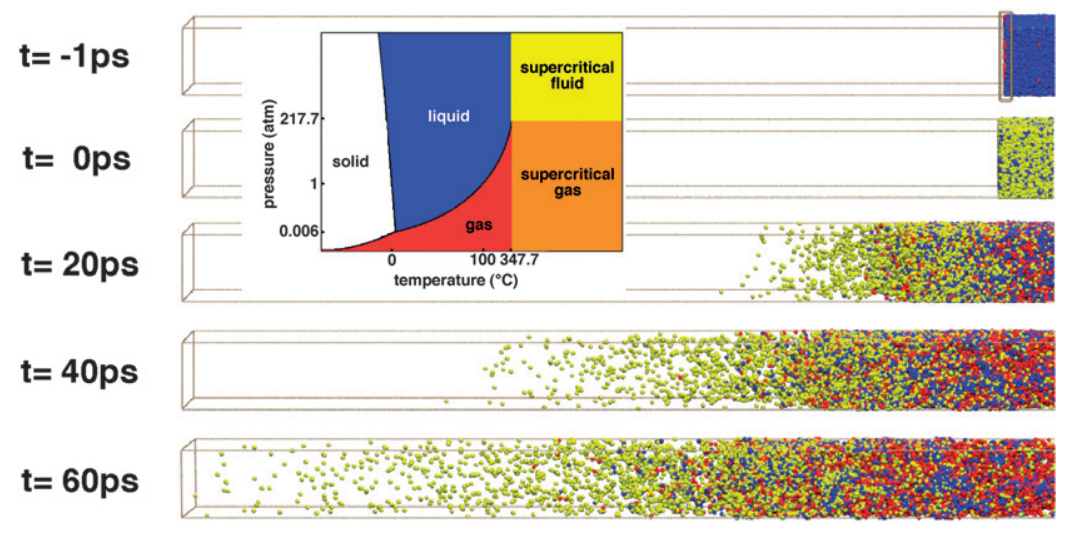

Fig. 5 Snaphots of the left side of the simulation box (total dimension $12 \times 12 \times 500 \mathrm{~nm}$ : shown $12 \times 12 \times 130 \mathrm{~nm}$ ) with approx. 140000 water molecules for different times. The snapshot at $t=-1$ ps represents the equilibrated box at $300 \mathrm{~K}$ and the one at $t=0 \mathrm{ps}$ after the instantaneous heating-up process. The other snapshots are taken out of the simulation at constant energy and volume (NVE).

In order to shed light on the lifetimes of aggregates in the expanding overheated phase and the question whether such aggregates live long enough to be detected via ultrafast ESCA, we have calculated lifetimes of highly excited (high temperature) dimers, trimers and tetramers. Fig. 6 shows the distribution of lifetimes of dimers, trimers and tetramers for trajectories $(775 \mathrm{~K})$ for which dissociation could be observed (non- reactive trajectories were omitted). For the dimer (Fig. 6, upper panel) half of the dissociated aggregates have a lifetime smaller than $2 \mathrm{ps}$. The trimer and tetramer (Fig. 6, middle and lower panels) show lifetime distributions which are shifted to longer lifetimes. Only $20 \%$ of the dissociated trimer has a lifetime smaller than 2 ps and only $10 \%$ in the case of the tetramer. The remaining trajectories of the trimer and the tetramer are distributed over a wide range compared to the dimer and in the case of the tetramer even $30 \%$ of the trajectories exhibit an aggregate lifetime larger than 24 ps. In addition, the fraction of nondissociating trajectories increases for increasing aggregate size.

These results show that aggregates at supercritical conditions (starting initially in their minimum-energy configurations) have predicted lifetimes on the order of a few to tens of picoseconds, depending critically on the size of the aggregates. Furthermore, it is expected that larger aggregates consisting of 5-20 molecules exhibit even larger lifetimes and there should be no problems in detecting them in the present type of experiment. Because the initial conditions of the simulation may not necessarily reflect the real conditions in the experiment (in terms of aggregate formation) the lifetimes obtained may be regarded as rough estimates rather than precise predictions.

The current results strongly suggest that the phase evolution of superheated water is not necessarily a trivial process starting from the hot water phase and leading rapidly to isolated molecules in the gas phase. Depending upon the initial temperature a fast "normal evaporation" or a dispersion-like evolution of the phase may be observed in real-time with partial large heterogeneities in the aggregate size. In the future we aim to shed more light onto this phenomenon of transient heterogeneity in an expanding supercritical (hydrogen-bonded) phase which may be special for water and which may change for other systems depending upon the hydrogen-bonding interactions.

\section{Conclusions and outlook}

In summary, we have demonstrated that ultrafast electronic spectroscopy for chemical analysis (ESCA) near liquid interfaces (being conceptually an ultrafast extreme 

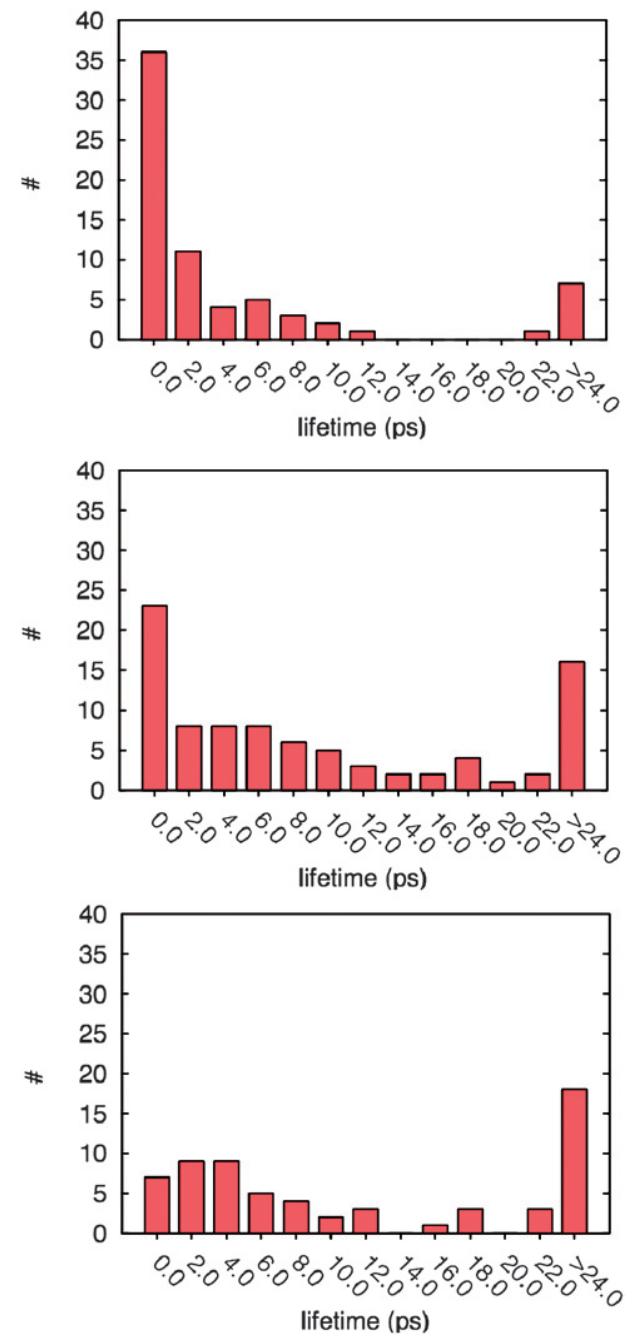

Fig. 6 Distribution of lifetimes of the dissociated aggregates (starting at minimum energy configuration). Upper panel: dimer (number (\#) of dissociated aggregates: 70 out of 101). Middle panel: trimer (number of dissociated aggregates: 88 out of 101). Lower panel: tetramer (number of dissociated aggregates: 64 out of 101).

ultraviolet photoelectron spectroscopy) is a powerful new two-dimensional spectroscopic tool that can monitor electron orbital energies and chemical shifts in time in the condensed phase near liquid interfaces, and that it enables a new avenue for monitoring ultrafast processes in chemistry and biologic model systems near water and other liquid interfaces. In a first application of the technique, we have shed light onto the dynamics and evolution of superheated and supercritical water phases and related phase transitions which are interesting both from a fundamental point of view and for certain analytical applications. ${ }^{44} \mathrm{With}$ regard to the evolution of superheated phases we found that the ultrafast process is not necessarily a trivial one starting from the metastable phase and leading directly to isolated molecules. The features of the evolution appear to be governed by hydrogen-bond dynamics and energetics.

Together with theory, the main future goal of our group will be to develop the current technique into an ultrafast photoemission spectroscopy (in the spirit of 
liquid interface ESCA, but going much beyond), namely to trace molecular orbitals (and their autocorrelation functions) during molecular transformations near liquid interfaces. One of the main challenges will be the clear distinction of liquid surface phenomena and processes in the bulk (below the first mono layer), which could in principle be addressed in EUV wavelength-dependent experiments (due to different probing and electron escape depths).

\section{Acknowledgements}

This work was supported by the Deutsche Forschungsgemeinschaft (SPP1134, "Investigation of Transient Structures of Photo-Induced Chemical Reactions in Solution with Ultrafast XUV-Photoelectron Spectroscopy", Project AB63/7-1,2, SFB 755 "Nanoscale Photonic Imaging", Project B2, GK782 "Dynamics and spectroscopy of molecular aggregates, coils and networks"), the Fonds der Chemischen Industrie and the Volkswagenstiftung. The joint research project was also financially supported by the State of Lower Saxony and the Volkswagen Foundation, Hannover, Germany.

\section{References}

1 H. E. Stanley, Introduction to Phase Transitions and Critical Phenomena, Oxford University Press, Oxford, 1971.

2 P. G. Debenedetti, Metastable Liquids: Concepts and Principles, Princeton Academic Press, Princeton, New Jersey, 1996.

3 E. Vöhringer-Martinez, B. Hansmann, H. Hernandez-Soto, H. Hernandez, J. S. Francisco, J. Troe and B. Abel, Science, 2007, 315, 497-501.

4 B. L. de Groot and H. Grubmüller, Science, 2001, 294, 2353-2357.

5 B. Winter, E. F. Aziz, U. Hergenhahn, M. Faubel and I. V. Hertel, J. Chem. Phys., 2007, 126, 124504.

6 P. Wernet, D. Nordlund, U. Bergmann, M. Cavalleri, M. Odelius, H. Ogasawara, L. A. Nyslund, T. K. Hirsch, L. Ojambe, P. Glatzel, L. G. Pettersson and A. Nilsson, Science, 2004, 304, 995-999.

7 Z. Wang, Y. Pang and D. D. Dlott, J. Phys. Chem. A, 2007, 111, 3196-3208.

8 S. Ashihara, N. Huse, A. Espagne, E. T. Nibbering and T. Elsaesser, J. Phys. Chem. A, 2007, 111, 743-746.

9 M. L. Cowan, B. D. Bruner, N. Huse, J. R. Dwyer, B. Chugh, E. T. Nibbering, T. Elsaesser and R. J. Miller, Nature, 2005, 434, 199-202.

10 S. Woutersen and H. J. Bakker, Phys. Rev. Lett., 2006, 96, 138305.

11 A. F. Goncharov, N. Goldmann, L. E. Fried, C. Crowhurst, I.-F. Kuo, W. C. J. Mundy and J. M. Zaug, Phys. Rev. Lett., 2005, 94, 125508.

12 F. Bencivenga, A. Cunsolo, M. Krisch, G. Monaco, G. Ruocco and F. Sette, Phys. Rev. E, 2007, 75, 051202.

13 A. Charvat, B. Stasicki and B. Abel, J. Phys. Chem. A, 2006, 110, 3297-3306.

14 N. Goldmann and L. E. Fried, J. Chem. Phys., 2007, 126, 134505.

15 N. Akiya and P. E. Savage, Chem. Rev., 2002, 102, 2725.

16 L. J. Rothschild and R. L. Mancinelli, Nature, 2001, 409, 1092-1101.

17 T. B. Brill, J. Phys. Chem. A, 2000, 104, 4343.

18 B. Winter and M. Faubel, Chem. Rev., 2006, 106, 1176-1211.

19 P. Agostini and L. F. DiMauro, Rep. Prog. Phys., 2004, 67, 813.

20 M. Drescher, Z. Phys. Chem., 2004, 218, 1147-1168.

21 U. Emmerichs, S. Woutersen and H. J. Bakker, J. Opt. Soc. Am., 1997, 14, 1480.

22 M. Ferray, A. L. L' Huillier, X. F. Li, L. A. Lompre and G. Mainfray, Phys. Rev. A, 1989, 39, 5751.

23 E. Seres, J. Seres, F. Krausz and C. Spielmann, Phys. Rev. Lett., 2004, 92, 163002.

24 Coherent Sources of XUV Radiation, ed. P. Jaegle, Springer, New York, 2006.

25 M. Faubel, in Adv. Series in Physical Chemistry, ed. C. Y. Ng, World Scientific, New York, 2000.

26 M. Faubel and T. Kisters, Nature, 1989, 339, 527-529.

27 M. Faubel, S. Schlemmer and J. P. Toennies, Z. Phys. D: At., Mol. Clusters, 1988, 10, 269.

28 J. Jasny, U. Teubner, W. Theobald, C. Wäulker, J. Bergmann and F. P. Schäfer, Rev. Sci. Instrum., 1994, 65, 1631. 
29 M. Hentschel, R. Kienberger, C. Spielmann, G. A. Reider, N. Milosevic, T. Brabec, P. Corkum, U. Heinzmann, M. Drescher and F. Krausz, Nature, 2001, 414, 509-513.

30 H. J. C. Berendsen, D. van der Spoel and R. van Drunen, Comput. Phys. Commun., 1995, 91, 43-56.

31 E. Lindal, B. Hess and D. van der Spool, J. Mol. Model., 2001, 7, 306-317.

32 B. Guilliot, J. Mol. Liq., 2002, 101, 219-260.

33 W. L. Jorgensen, J. Chandrasekar, J. D. Madura, R. W. Impey and M. Klein, J. Chem. Phys., 1983, 79, 926-935.

34 M. W. Schmidt, K. K. Baldridge, J. A. Boatz, S. T. Elbert, M. S. Gordon, J. H. Jensen, S. Koseki, N. MAtsunaga, K. A. Nguyen, S. Su, T. L. Windus, M. Dupois and J. A. Montgomery, J. Comput. Chem., 1993, 14, 1347-1363.

35 A. Charvat, E. Lugovoj, M. Faubel and B. Abel, Rev. Sci. Instrum., 2004, 75, 1209.

36 M. Faubel, B. Steiner and J. P. Toennies, J. Chem. Phys., 1997, 106, 9013-9031.

37 Y. Itikawa and N. Mason, J. Phys. Chem. Ref. Data, 2005, 34, 1-22.

38 H. Agren and V. Carravetta, Mol. Phys., 1985, 55, 901.

39 Y. Lim, T. Westerwalbesloh, A. Aschentrup, A. Wehmeyer, G. Haindl, U. Kleineberg and U. Heizmann, Appl. Phys. A, 2001, 72, 121.

40 L. Nugent-Glandorf, M. Scheer, D. A. Samuels, A. M. Mulhisen, E. R. Grant, X. Yang, V. M. Bierbaum and S. R. Leone, Phys. Rev. Lett., 2001, 87, 193002.

41 L. Belau, K. R. Wilson, S. R. Leone and M. Ahmed, J. Phys. Chem. A, 2007, 111, $10075-$ 10083.

42 O. Björnholm, F. Federmann, S. Kakar and T. Moller, J. Chem. Phys., 1999, 111, 546-550.

43 C. A. Jeffery and P. H. Austin, J. Chem. Phys., 1999, 110, 484496.

44 A. Charvat and B. Abel, Phys. Chem. Chem. Phys., 2007, 9, 3335-3360. 